Calidad de vida relacionada con la salud y Hemoglobina Glicosilada en pacientes con Diabetes Mellitus tipo 2

\title{
Health-related quality of life and glycosylated hemoglobin in patients with type 2 diabetes mellitus
}

https://doi.org/10.37135/ee.04.10.01

\author{
Autores \\ Byron Eduardo Coello-Viñán ${ }^{1,2}$ - https://orcid.org/0000-0002-4396-8746 \\ Jenny Elizabeth Coello-Viñán ${ }^{1,3}$ - https://orcid.org/0000-0002-2456-659X \\ Mayra Elizabeth Sánchez De la Torre ${ }^{1,3}$ - https://orcid.org/0000-0001-7040-8499 \\ ${ }^{1}$ Escuela Superior Politécnica de Chimborazo, Riobamba, Ecuador \\ ${ }^{2}$ Centro de Salud tipo C Espoch - Lizarzaburu, Dirección distrital 06D01, Riobamba, Ecuador \\ ${ }^{3}$ Centro de Salud tipo B Chambo, Dirección distrital 06D01, Chambo, Ecuador
}

Autor de correspondencia: Dr. Byron Eduardo Coello Viñán. Centro de Salud tipo C Espoch Lizarzaburu, Av. Mons. Leonidas Proaño y Atabasco, Riobamba, Ecuador; código postal ECU060101; correo elctrónico: taiomed@hotmail.com; Teléfono: 0987043593

\section{RESUMEN}

La diabetes mellitus afecta negativamente la calidad de vida de quienes la padecen; al respecto, se realizó un estudio no experimental de corte transversal, con el objetivo de determinar la relación entre calidad de vida según las dimensiones del cuestionario Euro-Qol (EQ-5D) y los valores de HbA1c 42 en pacientes con diagnóstico de diabetes mellitus tipo 2 del barrio El Retamal de la ciudad de Riobamba entre los meses de enero y febrero de 2019. Los datos sobre calidad de vida relacionada con la salud se recolectaron mediante el cuestionario EQ-5D, mientras que los valores de hemoglobina glicosilada se obtuvieron a partir de las historias clínicas. A través de una regresión lineal univariante, se estableció la relación estadísticamente significativa entre todas las dimensiones del cuestionario EQ-5D y los valores de hemoglobina glicosilada con un valor de $\mathrm{p}<0,001$; lo que permitió concluir que la autopercepción de la calidad de vida relacionada con la salud y los valores de hemoglobina glicosilada guardan una estrecha relación.

Palabras clave: diabetes mellitus, hemoglobina A glucada, calidad de vida. 


\begin{abstract}
Diabetes mellitus negatively affects the quality of life of patients who suffer it. In this regard, a non-experimental cross-sectional study was carried out in order to determine the relationship between quality of life according to the dimensions of the Euro-Qol (EQ-5D) questionnaire and the HbA1c 42 values in patients diagnosed with type 2diabetes mellitus in El Retamal neighborhood, city of Riobamba from January to February 2019. Data about health-related quality of life were collected using the EQ-5D questionnaire, while glycosylated hemoglobin values were obtained from the medical records. The statistically significant relationship was established between all the dimensions of the EQ-5D questionnaire and the glycosylated hemoglobin values with a value of $p<0.001$ through a univariate linear regression. It was concluded that self-perception of health-related quality of life and glycosylated hemoglobin values are closely related
\end{abstract}

Keywords: Diabetes Mellitus, Glycated Hemoglobin A, Quality of Life.

\title{
INTRODUCCIÓN
}

La Diabetes Mellitus (DM) es una enfermedad crónica y compleja, ${ }^{(1,2)}$ identificada por la Organización Mundial de la Salud (OMS) como una de las cuatro enfermedades no transmisibles (ENT) que requieren intervención prioritaria. ${ }^{(3)}$

Según estimaciones de la OMS, en 2014, cerca de 422 millones de adultos a nivel mundial padecían diabetes, casi cuatro veces más que en 1980 (108 millones). ${ }^{(3)}$ Esta patología es responsable de la muerte de alrededor de 5 millones de personas y se calcula un costo relacionado con su enfrentamiento de entre 673.000 millones y 1.197 .000 millones de dólares estadounidenses. Se prevé que podrían existir 642 millones de personas viviendo con esa enfermedad para el año $2040 . .^{(4)}$

Los datos epidemiológicos indican que la diabetes se encuentra en niveles críticos y tendencia al aumento. ${ }^{(5-7)}$ Cada siete segundos, muere alguna persona que la padece, representando unas cuatro millones de muertes anualmente. ${ }^{(5)}$

El entorno ecuatoriano no escapa a esa realidad, según la Encuesta Nacional de Salud y Nutrición 2011-2013 (ENSANUT), la prevalencia de diabetes en esa población con edades comprendidas entre 10 y 59 años es de $2.7 \%$, calculándose que uno por cada diez ecuatorianos padece esa enfermedad. ${ }^{(8)}$

Diversos aspectos de esa patología, tales como: morbilidad prematura, mortalidad, esperanza de vida reducida, necesidad de modificar estilos de vida, uso habitual de medicamentos prolonga- 
damente, además de otros; tienen la potencialidad para afectar la calidad de vida relacionada con la salud (CVRS) de los afectados. ${ }^{(9-11)}$

La CVRS depende de la percepción del individuo acerca de la satisfacción con respecto a los dominios del estado de salud que se vean afectados. ${ }^{(12)}$ Actualmente, la comunidad científica presta mucha atención a su evaluación, a partir de un concepto relacionado con la noción de bienestar en las esferas física, psicológica, socioeconómica y cultural.(13)

Diversos estudios muestran un deterioro de la CVRS en pacientes diabéticos al compararlos con individuos sanos de similares características sociodemográficas. ${ }^{(14-16)}$ Sin embargo, no existe claridad con respecto a la relación entre los valores de Hemoglobina Glicosilada (HbA1c) y la CVRS en este tipo de enfermo; lo que cobra importancia cuando se busca aminorar el impacto de la patología.

La investigación que se presenta tuvo como objetivo determinar la relación entre calidad de vida según las dimensiones del cuestionario Euro-Qol (EQ-5D) y los valores de HbA1c en pacientes con diagnóstico de diabetes mellitus tipo 2.

\section{MATERIAL Y MÉTODOS}

Una investigación de tipo no experimental de corte transversal se desarrolló a partir del estudio de los datos de 42 pacientes mayores de 20 años, con diagnóstico de Diabetes Mellitus tipo 2 y un resultado de HbA1c de los últimos 3 meses, además de ser residentes regulares en el barrio El Retamal de la ciudad de Riobamba durante los meses de enero y febrero de 2019, excluyendo a aquellos que no podían o querían completar el cuestionario.

La CVRS se estableció mediante el cuestionario Euro-Qol (EQ-5D), el que comprende cinco dimensiones: movilidad, autocuidado, actividades habituales, dolor/malestar y ansiedad/depresión. El cuestionario fue aplicado a pacientes que acudieron a consulta de control en el centro de salud tipo C Espoch - Lizarzaburu. Las variables sociodemográficas: sexo, edad, peso y talla, así como el valor de la $\mathrm{HbA} 1 \mathrm{c}$ fueron tomados directamente de las historias clínicas individuales correspondientes.

La escala utilizada para el estudio de las dimensiones del cuestionario EQ-5D fue dicotómica nominal: sin problemas y con problemas. Los valores recolectados se organizaron en una base de datos en el programa Microsoft Excel versión 16.22 para Mac; mientras que, el análisis estadístico se hizo empleando el programa IBM SPSS Statistics versión 25 para Mac. 
La normalidad en la distribución de los datos se estableció mediante la prueba de Shapiro-Wilk; además se emplearon estadísticas descriptivas que incluyeron medias, desviaciones estándar, asimetrías y análisis de frecuencias. La relación entre los valores de Hb1Ac y las dimensiones de la CVRS se determinó mediante la prueba de $t$ de student y posteriormente con modelos de regresión lineal univariantes (ANCOVA), ajustando los análisis para las covariables edad e índice de masa corporal, consideradas como posibles factores de confusión.

Cumpliendo con las normas establecidas en la Declaración de Helsisnsky, los investigadores aplicaron un formulario de consentimiento informado a todos los participantes, se respetó la confidencialidad de la información individual del paciente y se obtuvieron las correspondientes autorizaciones en la entidad de salud involucrada en el estudio.

\section{RESULTADOS}

El estudio incluyó un total de 42 pacientes con diagnóstico de diabetes mellitus tipo 2, predominando el género femenino (59,50\%), con una media de edad de 58,38 \pm 9,66 años (rango: 42 83 años); la mayoría se agrupó entre 51 y 60 años $(45,20 \%)$ (tabla 1$)$.

La media del IMC fue de 28,09 $\pm 4,17 \mathrm{Kg} / \mathrm{m} 2$, lo que se correspondió con la preponderancia de pacientes con sobrepeso $(42,90 \%)$, sin que se encontraran pacientes con bajo peso. En cuanto a los valores de HbA1c, predominaron los menores de 7, con un promedio de 7,31 $\pm 4,17$ (tabla 1).

Tabla 1. Distribución de los pacientes según características generales y clínicas

\begin{tabular}{|c|c|c|c|c|}
\hline & $\mathbf{N}$ & $\%$ & Media (SD) & Asimetría \\
\hline Sexo Masculino & 17 & 40,50 & & \\
\hline Femenino & 25 & 59,50 & & \\
\hline Edad (años) & & & $58,38(9,66)$ & 0,44 \\
\hline$\leq 50$ & 7 & 16,70 & & \\
\hline $51-60$ & 19 & 45,20 & & \\
\hline $61-70$ & 11 & 26,20 & & \\
\hline$\geq 71$ & 5 & 11,90 & & \\
\hline Peso (Kg) & & & $69,74(11,81)$ & $-0,65$ \\
\hline Talla (m) & & & $1,57(0,07)$ & $-0,60$ \\
\hline IMC $\left(\mathrm{Kg} / \mathrm{m}^{2}\right)$ & & & $28,09(4,17)$ & $-0,08$ \\
\hline Normal & 11 & 26,20 & & \\
\hline Sobrepeso & 18 & 42,90 & & \\
\hline Obesidad & 13 & 31,00 & & \\
\hline Hb1Ac (\%) & & & $7,31(1,16)$ & 0,82 \\
\hline$<7$ & 19 & 45,20 & & \\
\hline $7-9$ & 17 & 40,50 & & \\
\hline$>9$ & 6 & 14,30 & & \\
\hline
\end{tabular}

Abreviaciones: SD: Desviación estándar; IMC: índice de masa corporal. 
En relación con las dimensiones de la CVRS, la gran mayoría de los pacientes refirió tener problemas de ansiedad y depresión (59,50\%); sin embargo, en relación con la movilidad, cuidado personal y actividades habituales prevalecieron los que no declararon dificultades (tabla 2).

Tabla 2. Distribución según dimensiones de la calidad de vida relacionada con la salud

\begin{tabular}{lcccc}
\hline & \multicolumn{2}{c}{ Sin problemas } & \multicolumn{2}{c}{ Con problemas } \\
\hline & N & \% & N & $\%$ \\
Movilidad & 33 & 78,60 & 9 & 21,40 \\
Cuidado personal & 33 & 78,60 & 9 & 21,40 \\
Actividades habituales & 35 & 83,30 & 7 & 16,70 \\
Dolor/malestar & 21 & 50,00 & 21 & 50,00 \\
Ansiedad depresión & 17 & 40,50 & 25 & 59,50 \\
\hline
\end{tabular}

La prueba t de student posibilitó establecer la relación entre la CVRS y los valores de Hb1Ac; al respecto, se encontró significación estadística en cuanto a las dimensiones de dolor/malestar y ansiedad/depresión con un valor de p de 0,001 y $<0,001$ respectivamente (tabla 3 ).

Tabla 3. Relación entre la CVRS y HbA1c

\begin{tabular}{lcccc}
\hline \multicolumn{1}{c}{ CVRS } & Media & SD & p valor & p valor* \\
\hline Movilidad & & & & \\
Sin problemas & 7,25 & 1,16 & 0,546 & $<\mathbf{0 , 0 0 1}$ \\
$\begin{array}{l}\text { Con problemas } \\
\text { Cuidado personal }\end{array}$ & 7,52 & 1,20 & & \\
$\begin{array}{l}\text { Sin problemas } \\
\text { Con problemas }\end{array}$ & 7,19 & 1,12 & 0,211 & $<\mathbf{0 , 0 0 1}$ \\
Actividades habituales & 7,74 & 1,26 & & \\
Sin problemas & 7,22 & 1,09 & & \\
$\begin{array}{l}\text { Con problemas } \\
\text { Dolor/Malestar }\end{array}$ & 7,78 & 1,43 & 0,256 & $<\mathbf{0 , 0 0 1}$ \\
$\begin{array}{l}\text { Sin problemas } \\
\text { Con problemas }\end{array}$ & 6,76 & 0,84 & & \\
Ansiedad/Depresión & 7,87 & 1,18 & $\mathbf{0 , 0 0 1}$ & $<\mathbf{0 , 0 0 1}$ \\
$\begin{array}{l}\text { Sin problemas } \\
\text { Con problemas }\end{array}$ & 6,43 & 0,51 & & \\
\hline
\end{tabular}

*Modelo ajustado por edad e IMC

Los análisis ajustados para las covariables edad e IMC, mediante modelos de regresión lineal univariantes (ANCOVA), encontrándose relación estadísticamente significativa entre todas las dimensiones de la CVRS y los valores de HbA1c con un valor de $\mathrm{p}<0,001$ (tabla 3 ). 


\section{DISCUSIÓN}

Los valores de índice de masa corporal hallados sugieren hábitos alimenticios inadecuados en la mayoría de la población estudiada. Fisiopatológicamente, existe un vínculo estrecho entre sobrepeso, obesidad y diabetes mellitus que ha sido corroborado por varios estudios realizados, ${ }^{(17-19)}$ esa relación permitió acuñar el término "diabesity" ${ }^{(20-25)}$ para referirse a la presencia de estas en un mismo paciente.

Diversos investigadores establecen la existencia de un deterioro de la autopercepción de la calidad de vida en pacientes con enfermedades crónicas como la diabetes mellitus. ${ }^{(26-28)} \mathrm{Al}$ respecto, en la investigación que se presenta los problemas en la dimensión de ansiedad/depresión fueron los únicos que predominaron; mientras que, en el resto de los factores explorados prevalecieron los sujetos que consideraron no existir dificultades. Ese resultado indica que la mayor parte los participantes aún conservan un buen nivel de independencia y capacidad para desarrollar actividades básicas. Varios autores señalan que la vida bajo el padecimiento de una enfermedad crónica constituye una casusa para sufrir trastornos por ansiedad y depresión. ${ }^{(29-31)}$

El análisis mediante la prueba ANCOVA, considerando el peso y el IMC como variables confusoras, posibilitó establecer una relación estadísticamente significativa entre las dimensiones del cuestionario EQ-5D y los valores de hemoglobina glicosilada. Esos resultados concuerdan con los obtenidos en estudios similares al respecto. ${ }^{(10,11,15,32-35)}$

\section{CONCLUSIONES}

Los resultados del estudio permitieron establecer la existencia de relación entre la autopercepción de la calidad de vida relacionada con la salud y los valores de hemoglobina glicosilada en pacientes con diabetes mellitus tipo 2; lo que apunta hacia la necesidad de velar por un adecuado control metabólico en los pacientes aquejados de esa patología.

Conflictos de intereses: los autores declaran que no existen.

\section{Declaración de contribución:}

Byron Eduardo Coello Viñán, Jenny Elizabeth Coello Viñán y Mayra Elizabeth Sánchez De la Torre realizaron el proceso investigativo y redacción del artículo científico. 


\section{REFERENCIAS BIBLIOGRÁFICAS}

1. Schanner C, Falck A, Keskitalo A, Hautala N. Health-related quality of life of Finnish patients with diabetes. Scand J Public Health [Internet]. 2016 [citado 12 Feb 2019]; 44(8): 765-771. Disponible en: https://doi.org/10.1177/1403494816666732.

2. American Diabetes Association. Standards of Medical Care in Diabetes 2019. Diab Care [Internet]. 2019 [citado 12 Feb 2019]; 42(1). Disponible en http://care.diabetesjournals. org/content/diacare/suppl/2018/12/17/42.Supplement_1.DC1/DC_42_S1_Combined_ FINAL.pdf.

3. World Health organization. Global Report of Diabetes [Internet]. Ginebra: OMS; 2016 [citado 12 Feb 2019]. Disponible en: https://apps.who.int/iris/bitstream/handle/ 10665/204871/9789241565257_eng.pdf?sequence=1.

4. International Diabetes Federation. Altas de Diabetes de la FID [Internet]. Bélgica: Karakas Print; 2015 [citado 12 Feb 2019]. Disponible en: https://www.fundaciondiabetes. org/upload/publicaciones_ficheros/95/IDF_Atlas_2015_SP_WEB_oct2016.pdf.

5. International Diabetes Federation. Global Diabetes Plan 2011 - 2021 [Internet]. Belgium: IDF; 2015 [citado 12 Feb 2019]. Disponible en: https://idf.org/our.../129-global-diabetesplan-2011-2021.html.

6. Yisahak SF, Beagley J, Hambleton IR, et al. IDF diabetes atlas: diabetes in North America and the Caribbean: an update. Diabetes Res Clin Pract [Internet]. 2014 [citado 12 Feb 2019]; 103(2): 223-230. Disponible en: https://www.ncbi.nlm.nih.gov/pubmed/24321468.

7. Tamayo T, Rosenbauer J, Wild SH, Spijkerman AM, Baan C, Forouhi NG, et al. Diabetes in Europe: an update. Diabetes Res Clin Pract [Internet]. 2014 [citado 12 Feb 2019]; 103 (2): 206-217. Disponible en: https://www.ncbi.nlm.nih.gov/pubmed/24300019.

8. Ministerio de Salud pública del ecuador, Instituto Nacional de Estadística y Censos. Encuesta Nacional de Salud y Nutrición ENSANUT-ECU 2012 [Internet]. Guayaquil: El Telégrafo; 2014 [citado 13 Feb 2019]. Disponible en: http://www.ecuadorencifras.gob.ec/ documentos/web-inec/Estadisticas_Sociales/ENSANUT/MSP_ENSANUT-ECU_ 06-10-2014.pdf.

9. Gandhi N, Wareham, F. Epidemiology of diabetes. Medicine (Abingdon) [Internet]. 2014 [citado 13 Feb 2019]; 42(12): 698-702. Disponible en: https://www.ncbi.nlm.nih. gov/pmc/articles/PMC4282306/. 
10. López A, Cechetto E, Aguirre A, Ontiveros M, Roitter C, García A, et al. Factores asociados con la calidad de vida relacionada con la salud en personas con diabetes mellitus de la obra social universitaria de Cordoba. Rev Fac Cien Med Univ Nac Cordoba [Internet]. 2017 [citado 13 Feb 2019]; 74(4): 306-312. Disponible en: https://revistas.unc. edu.ar/index.php/med/article/view/15835/19446.

11. Kamradt M, Krisam J, Kiel M, Qreini M, Besier W, Szecsenyi J, et al. Health-Related Quality of Life in Primary Care: Which Aspects Matter in Multimorbid Patients with Type 2 Diabetes Mellitus in a Community Setting?. PLoS One [Internet]. 2017 [citado 13 Feb 2019]; 12(1): 1-14. Disponible en: https:/www.ncbi.nlm.nih.gov/pmc/articles/ PMC5268781/.

12. Karimi M, Brazier J. Health, Health-Related Quality of Life, and Quality of Life: What is the Difference?. Pharmacoeconomics [Internet]. 2016 [citado 20 Feb 2019]; 34(7): 645-649. Disponible en: https://www.ncbi.nlm.nih.gov/pubmed/26892973.

13. Saleem F, Hassali MA, Shafie AA. A cross-sectional assessment of health-related quality of life (HRQoL) among hypertensive patients in Pakistan. Heal Expect [Internet]. 2014 [citado 20 Feb 2019]; 17(3): 388-395. Disponible en: https://www.ncbi.nlm.nih.gov/pmc/ articles/PMC5060735/pdf/HEX-17-388.pdf.

14. Vancampfort D, Mitchell AJ, De Hert M, Sienaert P, Probst M, Buys R, et al. Type 2 diabetes in patients with major depressive disorder: A Meta-Analysis of prevalence estimates and predictors. Depress Anxiety [Internet]. 2015 [citado 20 Feb 2019]; 32(10): 763-73. Disponible en: https://onlinelibrary.wiley.com/doi/pdf/10.1002/da.22387.

15. D’Souza MS, Venkatesaperumal R, Ruppert SD, Karkada SN, Jacob D. Health Related Quality of Life among Omani Men and Women with Type 2 Diabetes. J Diabetes Res [Internet]. 2016 [citado 20 Feb 2019]; 2016. Disponible en: https://www.ncbi.nlm.nih. gov/pmc/articles/PMC4670671/.

16. Grandy S, Sternhufvud C, Ryden A, Sugg J, Rohwedder K. Patient-reported outcomes among patients with type 2 diabetes mellitus treated with dapagliflozin in a triple-therapy regimen for 52 weeks. Diabetes, Obes Metab [Internet]. 2016 [citado 22 Feb 2019]; 18(3): 306-309. Disponible en: https://www.ncbi.nlm.nih.gov/pubmed/26537439.

17. Serván PR. Obesity and Diabetes Mellitus. Nutr Hosp [Internet]. 2013 [citado 24 Feb 2019]; 28(5): 138-143. Disponible en: http://www.nutricionhospitalaria.com/pdf/6929 .pdf. 
18. Peplow P V. Topical Issue: Acu-obesity and Diabetes. JAMS J Acupunct Meridian Stud [Internet]. 2016 [citado 24 Feb 2019]; 9(3): 107-108. Disponible en: http://dx.doi.org/ 10.1016/j.jams.2016.01.009.

19. Gallagher EJ, LeRoith D. Obesity and Diabetes: The Increased Risk of Cancer and Cancer-Related Mortality. Physiol Rev [Internet]. 2015 [citado 24 Feb 2019]; 95(3): 727-748. Disponible en: https://www.ncbi.nlm.nih.gov/pmc/articles/PMC4491542/.

20. Pappachan JM, Fernandez CJ, Chacko EC. Diabesity and antidiabetic drugs. Mol Aspects Med [Internet]. 2019 [citado 24 Feb 2019]; 66: 3-12. Disponible en: https://doi. org/10.1016/j.mam.2018.10.004.

21. Pappachan JM, Viswanath AK. Medical Management of Diabesity: Do We Have Realistic Targets? Curr Diab Rep [Internet]. 2017 [citado 24 Feb 2019]; 4(2017). Disponible en: http://dx.doi.org/10.1007/s11892-017-0828-9.

22. Farag YMK, Gaballa MR. Diabesity: An overview of a rising epidemic. Nephrol Dial Transplant [Internet]. 2011 [citado 24 Feb 2019]; 26(1): 28-35. Disponible en: https://a cademic.oup.com/ndt/article/26/1/28/1838186.

23. Rajeswaran C. Diabesity management worldwide. Futur Sci OA [Internet]. 2016 [citado 24 Feb 2019]; 2(1). Disponible en: https://www.ncbi.nlm.nih.gov/pmc/articles/PMC5137961/ pdf/fso-02-86.pdf.

24. Farzi A, Hassan AM, Zenz G, Holzer P. Diabesity and mood disorders: Multiple links through the microbiota-gut-brain axis. Mol Aspects Med [Internet]. 2019 [citado $24 \mathrm{Abr}$ 2019]; 66: 80-93. Disponible en: http://www.ncbi.nlm.nih.gov/pubmed/30513310.

25. Kalra S. Diabesity. J Pak Med Assoc [Internet]. 2013 [citado 24 Feb 2019]; 63(4): 532-534. Disponible en: https://jpma.org.pk/PdfDownload/4131.

26. Shiue I, Sand M. Quality of life in caregivers with and without chronic disease: Welsh Health Survey, 2013. J Public Heal (United Kingdom) [Internet]. 2017 [citado 27 Feb 2019]; 39(1): 34-44. Disponible en: https://academic.oup.com/jpubhealth/article/39/1/34/ 3065702.

27. Donnelly SC. Chronic disease and assessing quality of life? Qjm [Internet]. 2016 [citado 27 Feb 2019]; 109(11): 701-701. Disponible en: https://academic.oup.com/qjmed/article/ 109/11/701/2712511. 
28. Ojelabi AO, Graham Y, Haighton C, Ling J. A systematic review of the application of Wilson and Cleary health-related quality of life model in chronic diseases. Health Qual Life Outcomes [Internet]. 2017 [citado $27 \mathrm{Feb} 2019$ ]; 15: 1-15. Disponible en: https://www.ncbi.nlm.nih.gov/pmc/articles/PMC5725920/pdf/12955_2017_Article_ 818.pdf.

29. Sivertsen B, Johnson JA, Øverland S, Naicker K, Colman I, Skogen JC, et al. Symptoms of anxiety and depression in type 2 diabetes: Associations with clinical diabetes measures and self-management outcomes in the Norwegian HUNT study. Psychoneuroendocrinology [Internet]. 2017 [citado 27 Feb 2019]; 84: 116-123. Disponible en: http://dx. doi.org/10.1016/j.psyneuen.2017.07.002.

30. DeJean D, Giacomini M, Vanstone M, Brundisini F. Patient experiences of depression and anxiety with chronic disease: A systematic review and qualitative meta-synthesis. Ont Health Technol Assess Ser [Internet]. 2013 [citado 27 Feb 2019]; 13(16): 1-33. Disponible en: https://www.ncbi.nlm.nih.gov/pmc/articles/PMC3817854/pdf/ohtas-1333a.pdf.

31. Khambaty T, Callahan CM, Perkins AJ, Stewart JC. Depression and Anxiety Screens as Simultaneous Predictors of 10-Year Incidence of Diabetes Mellitus in Older Adults in Primary Care. J Am Geriatr Soc [Internet]. 2017 [citado 27 Feb 2019]; 65(2): 294-300. Disponible en: https://www.ncbi.nlm.nih.gov/pmc/articles/PMC5311025/pdf/nihms793339.pdf.

32. Rodríguez-Almagro J, García-Manzanares Á, Lucendo AJ, Hernández-Martínez A. Health-related quality of life in diabetes mellitus and its social, demographic and clinical determinants: A nationwide cross-sectional survey. J Clin Nurs [Internet]. 2018 [citado 27 Feb 2019]; 27(21-22): 4212-4223. Disponible en: https://www.ncbi.nlm.nih.gov/ pubmed/29987853.

33. Aro AK, Karjalainen M, Tiihonen M, Kautiainen H, Saltevo J, Haanpää M, et al. Glycemic control and health-related quality of life among older home-dwelling primary care patients with diabetes. Prim Care Diabetes [Internet]. 2017 [citado 27 Feb 2019]; 11(6): 577-582. Disponible en: http://dx.doi.org/10.1016/j.pcd.2017.07.001.

34. Dogan H, Harman E, Kocoglu H, Sargin G. Can metabolic control variables of diabetic patients predict their quality of life? J Am Soc Hypertens [Internet]. 2016 [citado 27 Feb 2019]; 10(1): 81-88. Disponible en: http://dx.doi.org/10.1016/j.jash.2015.11.014. 
35. Shim YT, Lee J, Toh MPHS, Tang WE, Ko Y. Health-related quality of life and glycae mic control in patients with Type 2 diabetes mellitus in Singapore. Diabet Med [Internet]. 2012 [citado 27 Feb 2019]; 29(8): 241-248. Disponible en: https://www.ncbi.nlm. nih.gov/pubmed/22507291.

Recibido: 7 de octubre de 2019

Aprobado: 4 de noviembre de 2020 